\title{
Study of the Epidemiology of Laryngeal Cancer in Sohag University Hospital
}

\author{
M. Abdel Kader 1, Al Hussien A A 2, M.NasrEldosoky 3 \\ 1- Professor of Otorhinolaryngology, Faculty of Medicine, Sohag University \\ 2- Lecturer of Otorhinolaryngology, Faculty of Medicine, Sohag University \\ 3-Resident of Otorhinolaryngology, Faculty of Medicine, Sohag University
}

\section{Abstract \\ Introduction}

Cancer larynx represents about1\%ofall malignancies and about 30\% of head and neck malignanciesand it is the second most common malignancy of the head and neck(1).

The typical patient is a man in his 50 s or 60 s with a history of smoking and/or alcohol use. However, the male predilection for this disease has recently decreased from a male: female ratio of 15: 1 to less than 5: 1 currently. This change in demographics has been attributed to increased rates of smoking among women and their increasing presence in equally toxic work environments (2).

Several risk factors have been reported, Smoking, Alcohol, gastroesophageal reflux, occupational exposure, and genetic factors. The most important one was smoking, Eighty-five percent of laryngeal cancers can be attributed to tobacco and alcohol use. Smoking is the predominant risk factor for laryngeal carcinoma, with alcohol use being an independent and synergistic effect (3). In terms of histopathology, 95\% to $98 \%$ of cancer of the larynx is of squamous cell origin (4). As far as pathogenesis is concerned, several predisposing factors have been reported, including smoking and alcohol consumption which are the most important ones. Other possible risk factors are exposure to carcinogens in the work environment, nutrition, viral
The purpose of this study is to present the epidemiological data of patients diagnosed with cancer of the larynx in Sohag University Hospital, Otorhinolaryngology department during the period from July 2013 till June 2015. At the same time, we review the current international literature and compare our results with those from similar series abroad.

\section{Materials and methods}

Epidemiological data were collected from those patients including personal information (age, sex, residence, occupation and special habits of medical importance (eg, alcohol and smoking including smoking types, index and activity).

The history taking including complaint, its duration and different symptoms encountered (dysphonia, dysphagia, neck swelling(s), stridor, dyspnea, referred otalgia and weight loss). In addition, general medical, oncologic and family history were also taken.

All patients were subjected to routine physical and otorhinolaryngologic examination including indirect and flexible fibreoptic laryngoscopy with description of the lesion, regional evaluation of the neck nodes and synchronous second primary tumor. Preoperative laboratory investigations and CT scan was performed for all patients for proper staging.

All patients in this study were subjected to direct laryngoscopies examination for accurate assessment 
and mapping of the site and extension of the primary tumor using different sized rigid laryngoscopies and all biopsies were taken for histopathological examination for microscopic examination and the histological grading of SCC was performed

\section{Results}

\section{Age of patients}

The average ages of our patients were 64.07 years with a range from 36 to 98 years, median 64years. About $66.6 \%$ of patients were above 60 years, $31.4 \%$ were at the age of 41-60 years, whereas only $2 \%$ were younger than 40 years of age with significant $P$ value (.02).

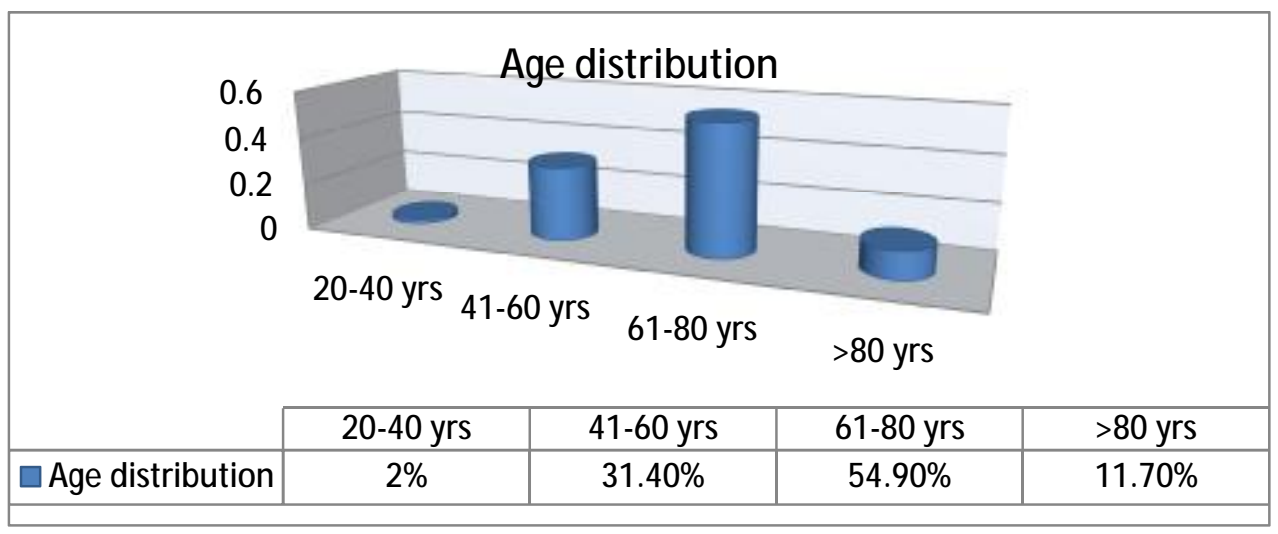

Figure (1) and table (1): Age distribution

\section{Gender}

The present study included a total number of 102 patients with laryngeal cancer during the period from July 2013 till June 2015. 97 patients (95.1\%) were males and 5 patients $(4.9 \%)$ were females.

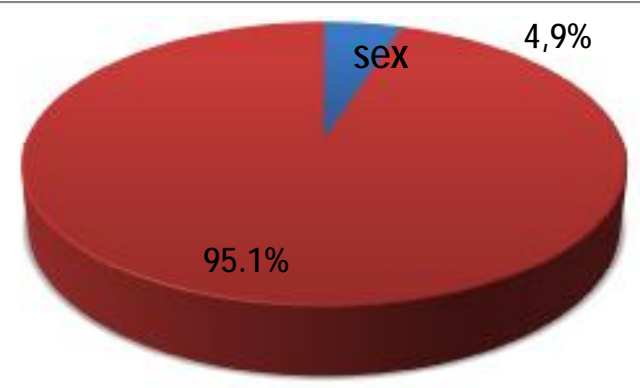

Female

Male

\begin{tabular}{|l|l|l|}
\hline Sex & Number & Percentage \\
\hline Female & 5 & $4.9 \%$ \\
\hline Male & 97 & $95.1 \%$ \\
\hline
\end{tabular}

Table (2) and Figure (2): Sex distribution 


\section{Occupation}

In terms of occupation, $49 \%$ of our patients were farmers, $11.8 \%$ were builders, $9.8 \%$ were carpenters, $8.8 \%$ were teachers, $4.9 \%$ were house wives, $4.9 \%$ were sellers, $3.9 \%$ were police men, $2.9 \%$ were moazen, $2 \%$ were plumbers, and $1 \%$ were accountant and lawyer of each one.

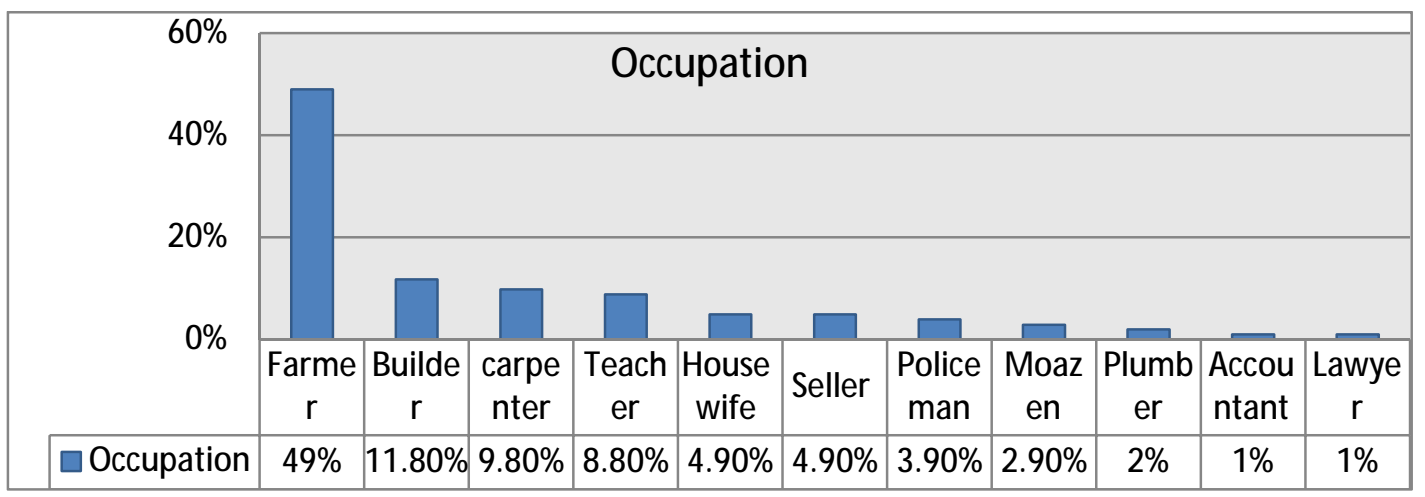

Table (3) and figure (3): Patient occupation

\section{Tobacco Use}

In total, $95.1 \%$ of our patients with cancer larynx were smokers while $4.9 \%$ were non smokers with significant $\mathrm{p}$ value (.oo4).

- More specifically, from the group of smokers, $81.4 \%$ of patients were active smokers, $13.7 \%$ were x-smokers, and only $4.9 \%$ were non smokers

In our study, among active smokers, $78.3 \%$ of patients were heavy smokers $(>200$ cigarette per year), $3.1 \%$ of patients were moderate smokers (100-200 cigarette per year).

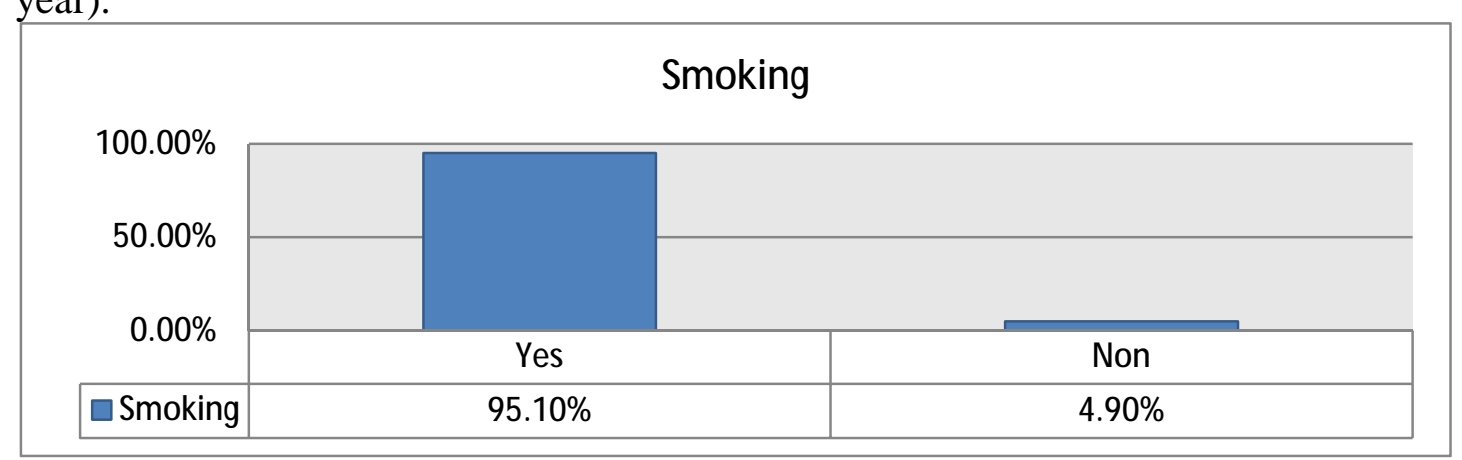

Figure (4) and table (4): Smoking incidence

\section{Alcohol Consumption}

Daily alcohol consumption was reported by 4 (3.9\%) of patients (mild users)., According to the US National Institute on Alcohol Abuse and Alcoholism (NIAAA) the definition for "heavy" alcohol use is over 5-6 drinks (12 grams of alcohol) per day for men and over 3-4 drinks per day for women. Of the remaining patients, $96.10 \%$ reported non alcohol consumption.

\section{Family History}

Negative family history in between patients with cancer larynx was reported. 


\section{Location}

Regarding the location of the tumor, we recorded $46(45.1 \%)$ cases of glottic neoplasm, $31(30.4 \%)$ of cases of transglottic neoplasm, $22(21.6 \%)$ of cases of supraglottic neoplasm and only $3(2.9 \%)$ of patients with subglottic neoplasm.

\section{Staging}

The staging of the tumor according to the TNM system were as follows: 14 patients $(13.7 \%)$ were found with Stage I, 9 patients $(8.9 \%)$ displayed Stage II, 57 patients $(55.9 \%)$ had Stage III and 22 patients $(21.5 \%)$ were presented with Stage IV.

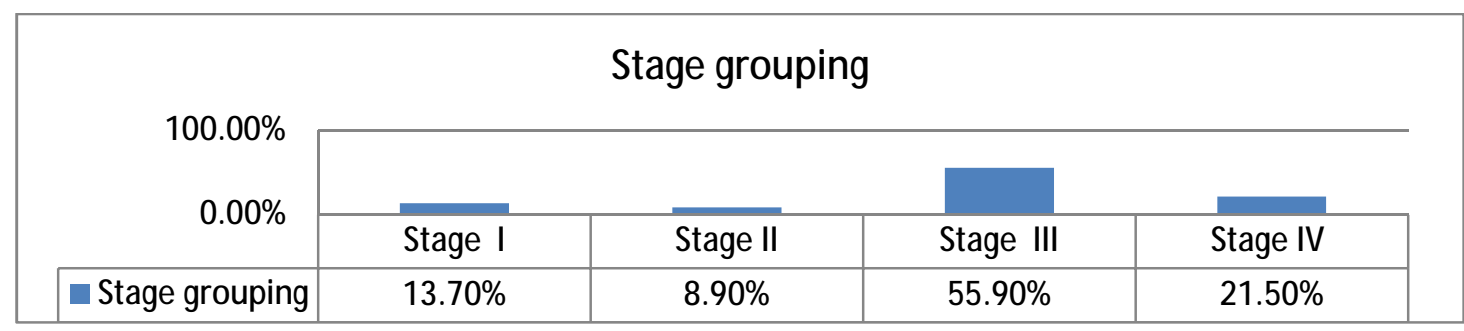

Figure (5) and Table (5): TNM staging of cancer larynx according to AJCC and UJCC

\section{Lymph Node Metastasis}

The presence of cervical lymph node metastasis was recorded in 34 patients as follow: 22 patients $(21.6 \%)$ were staged N1, $10(9.8 \%) \mathrm{N} 2$ and 2 patients $(1.9 \%) \mathrm{N} 3$.

\section{Differentiation}

Study results concerning histopathology of cancer larynx were as follows: 99 of all biopsies were SCC (97.10\%), and 3 of biopsies were verrucus type (2.90\%).

Study results concerning tumor differentiation of cancer larynx with SCC were as follows: 2 cases (2\%) were well differentiated (G1), 69 cases $(67.7 \%)$ were moderately differentiated (G2), 24 cases $(23.5 \%)$ were poorly differentiated (G3) and 4 cases $(3.9 \%)$ were undifferentiated $(\mathrm{G} 4)$.

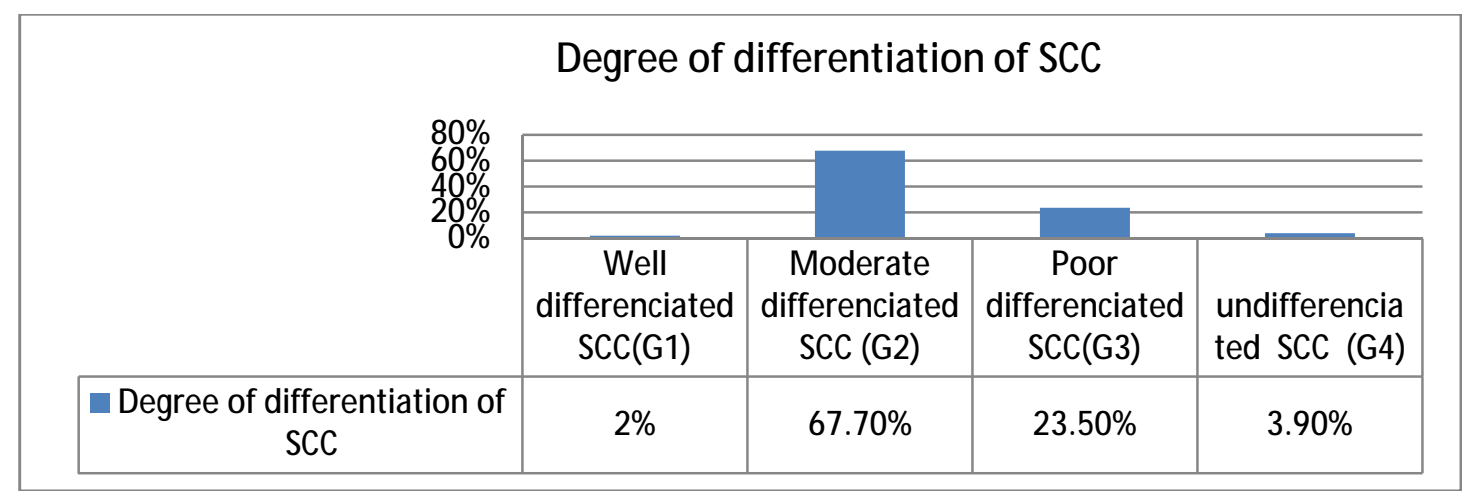

Figure (6) and Table (6): The degree of differentiation of SCC of patients with cancer larynx

\section{Distant metastasis}

Only 10 patients $(9.8 \%)$ were diagnosed with distant metastasis (M1) and the lung was the main site of secondary metastasis, additionally the presence of second primary neoplasms not established in our patients. 


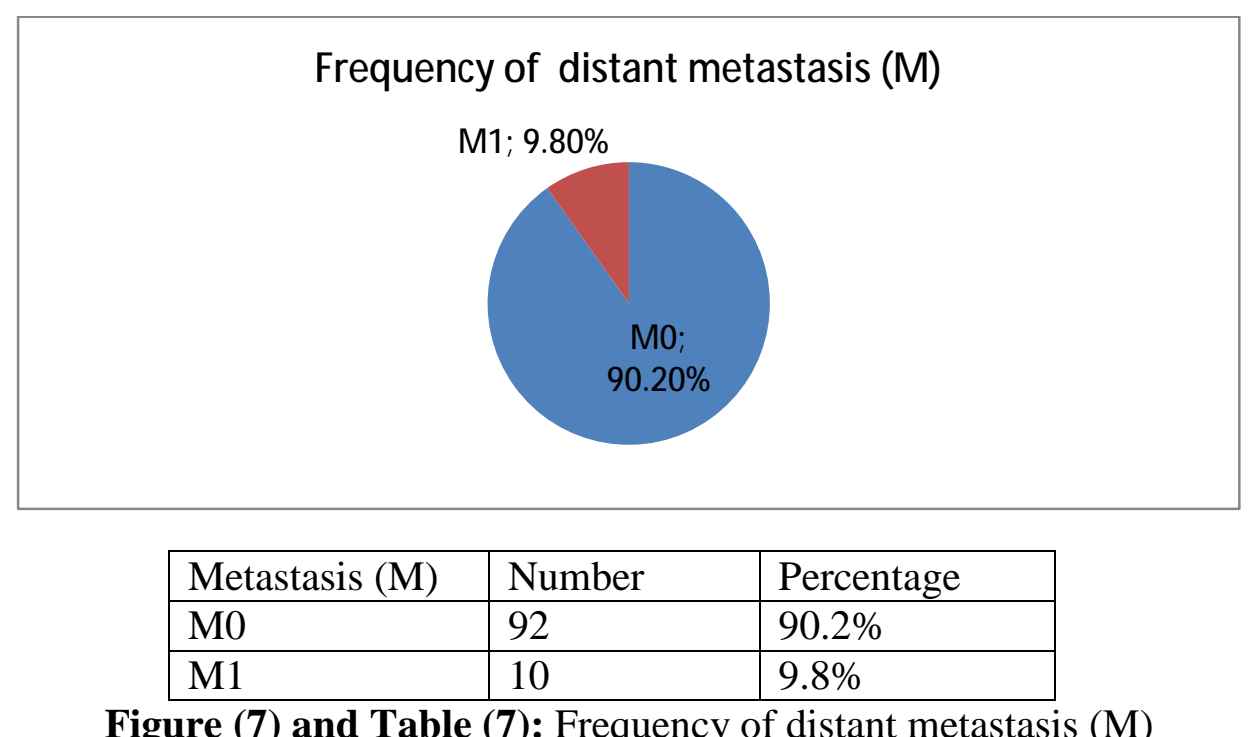

\section{Discussion}

The present study was carried out on 102 histopathologically confirmed laryngeal cancer patients who were admitted to our tertiary university hospital. The mean age was 64.07, ranging from 36 to 98 years. $54.9 \%$ of our patients were aged between 61-80 years old, $31.4 \%$ were aged between 41-60 years, $11.7 \%$ were aged above 80 years, and $2 \%$ were aged between 20-40 years. This means that the incidence of cancer larynx increased with age and it may affect younger age group. Laryngeal cancer is generally a disease of the elderly, with a peak incidence in the 50s and 60s (3). Most patients who developed this disease are males, and the maximum incidence occurs during the sixth decade of life (5).

In the present study, 97 patients were males and 5 patients were females. The male to female ratio was about 19.4:1, indicating a higher incidence among males. This is in line with study reported that cancer of the larynx is a predominantly male disease and the male to female ratio varies internationally from $30: 1$ to $5: 1$. In Europe, the ratio is $7: 1$. In recent years however, we have noted a significant increase in the percentage of women with laryngeal cancer(6-7).

Tobacco is evidently the most important risk factor for the development of laryngeal carcinoma. According to data from the international literature, smoking is associated with $95 \%$ of carcinomas of the larynx and is especially related to glottic cancer(8), This agreed withour study in which $95.1 \%$ of patients were smokers while $4.9 \%$ were non smokers, and this was particularly reported in cancer of the glottic region, then the transglottic and supraglottic regions respectively. In addition, the incidence among active smokers $(81.40 \%)$ was much higher than exsmokers $(13.70 \%)$. Our results were concomitant with study reported that, from a total of 1,088 patients with laryngeal cancer, $86.8 \%$ were smokers, $5.6 \%$ non-smokers and $7.6 \%$ exsmokers (9). In our study the incidence of cancer larynx in between non smokers' patients was very rare and this matched with the study ofTuyns et al.,(1988) concerning central Europe which showed that the risk for developing cancer of the larynx is 16 times greater in heavy smokers than in non-smokers. 
In our study $96.10 \%$ of patients were non alcoholic and this attributed to religion morals. Alcohol has been implicated as well as an important risk factor in the pathogenesis of laryngeal cancer. Indeed, the relation between alcohol consumption and the development of laryngeal carcinoma is proportional to the dose and duration of exposure (10). Chronic inflammation of the lining of the larynx from ethanol causes a series of mutations at gene level that disturb cell proliferation and promote carcinogenesis. A study reported that, in a total of 1088 patients with laryngeal cancer, daily alcohol consumption was observed in $43.2 \%$ of the patients(10). Alcohol consumption is mainly related to the development of cancer in the supraglottic part of the larynx and the hypopharynx. A german study reported that from all patients with cancer of the larynx, daily alcohol consumption was noted in $85.1 \%$, while another study showed that $76.3 \%$ of the patients consumed more than 25 gr of ethanol daily (11).

Smoking and alcohol consumption are considered to act synergistically. According to data from published studies, the reduction of tobacco and alcohol use will contribute to the prevention of cancer of the larynx and their cessation will reduce the disease by $90 \%$ (12).

Several epidemiological studies indicate a correlation between malignancy in the larynx and the socioconomic profile and occupation of patients. It is considered that the incidence of the disease is higher in lower-income and low educational level population. The majority of patients with cancer of the larynx are unemployed or unskilled workers and farmers without basic education (1314). In accordance with the above the majority of our patients were farmers $(49 \%)$ and unskilled workers $(23.6 \%)$, of low educational and socioeconomic level and this might be due to the geographic distribution of population referred to our hospital from Upper Egypt.

Cancer of the larynx has been associated with inhaled carcinogens in the workplace, but this is not completely documented as with other cancer locations in the head and neck area. The majority of patients with cancer of the larynx are workers in chemical industries and farmers. Cement dust, fertilizers, petroleum derivatives and polycyclic aromatic hydrocarbons, wood and metal dust, varnish etc have been implicated as risk factors at work environment (158-16).

In our study we did not find a specific type of occupational exposure or carcinogens with close relation to incidence of cancer larynx.

The relationship between GERD and laryngeal cancer is not clear; A study reported that GERD patients are risky to develop cancer larynx. On the contrary, Eva et al., (2007) did not find an intimate relationship. In our work, 54 patients had suggestive history for GERD including heartburn and regurgitation but no confirmatory diagnostic tests were conducted $(24 \mathrm{~h}$ $\mathrm{pH}$ monitoring)(17).

In our study there was no family history of cancer larynx and this was in line with the study that reported, no family history of laryngeal cancer(18). However, a genetic predisposition has been demonstrated in first degree relatives and it has been posited that the genetic foundations for this susceptibility is found in the DNA cell repair systems, in the efficacy of cell systems to metabolize carcinogens, and in control systems of the cell cycle or in a combination of all of these (19).

Significant differences are observed in the percentage of the anatomical position of the site of cancer in the 
larynx. The data of the international literature indicate the glottis as the most frequent location, while tumors found in the subglottis are considered extremely rare (6-7). In our study, the glottic region $(45.1 \%)$ was the commonest followed by transglottic (30.4\%), then supraglottic $(21.6 \%)$ and finally the subglottic region $(2.9 \%)$ being the least affected. These results were in line with a study carried out on 48 patients with laryngeal cancer and the results according to location were as follows: glottic in 30 patients, supraglottic localization in 14 patients, and only subglottic cancer in one patient(20). A study that carried out on 295 patients with SCC of the larynx and showed that the most common tumors were glottic-supraglottic (62.4\%), transglottis $(20.7 \%)$, glottic (10.8\%), supraglottic (5.1\%), and finally subglottic (1.0\%), which is a rare subsite for laryngeal cancer(21).

In our study $72.6 \%$ of cancer larynx was infilterating masses while $24.5 \%$ were cauliflower masses and $2.9 \%$ were ulcerative masses. All patients underwent endoscopic evaluation under general anesthesia and biopsies were taken for histopathological examination. SCC was found in $(97.10 \%)$ and verrucus type was found in $(2.90 \%)$, and this matched with studies that reported that SCC was found in $96.6 \%$ of patients(21). Also, about $85-95 \%$ of laryngeal malignancies are SCC (1).

It is also known that, depending on the degree of differentiation of cancer cells, carcinoma of the larynx is classified as highly, moderately and poorly differentiated. Tumors with lower differentiation have poorer prognosis, because of the greater risk of recurrence or metastasis (22-23). This is demonstrated in our study, by the fact that the neoplasms with good differentiation had a lower incidence of cervical metastasis compared with the tumors with low degree of differentiation. In our study $2 \%$ of patients with SCC of cancer larynx were well differentiated (G1), $67.7 \%$ were moderately differentiated (G2), $23.50 \%$ were poorly differentiated (G3) and $3.90 \%$ were undifferenciated (G4) (according to Broders classification).

In the present study, $\mathrm{T}$ classification was found to be as follows: T3 (61.7\%), T1 (16.7\%), T2 (11.8\%), and T4 (9.8\%). Also, the presence of cervical lymph node metastasis was recorded in 35 patients as follow: 22 patients $(21.6 \%)$ were staged $\mathrm{N} 1,10$ $(9.8 \%) \mathrm{N} 2$ and 2 patients $(1.9 \%) \mathrm{N} 3$. Also, distant metastasis reported in 10 patients $(9.8 \%)$ and the lung was the main site of secondary metastasis. TNM Staging was as follows: $55.9 \%$ were stage III, $21.5 \%$ were stage IV, $13.7 \%$ were stage I, and $8.9 \%$ were stage II, which indicates that patients presented in late stages of the disease. Our results were nearly similar to researchers who reported in their study that carried out on 295 patients with SCC of the larynx and showed that $\mathrm{T}$ classification was found to be as follows: T3 (66.1\%), T2 (12.8\%), T1 $(12.1 \%),(8.7 \%) \mathrm{T} 4$, and Tis $(0.3 \%)$. Also $61.9 \%$ of patients were N0, (36.0\%) were $\mathrm{N} 1,(1.7 \%)$ were $\mathrm{N} 2 \mathrm{a}$, and $(0.3 \%)$ were $\mathrm{N} 2 \mathrm{c}$ and no patient had distant metastasis. Staging was as follows: $68.5 \%$ were stage III, $12.1 \%$ were stage I, $11.1 \%$ were stage II, $5.9 \%$ were stage IVa, $2.1 \%$ were stage $\mathrm{IVb}$, and $0.3 \%$ were stage 0 (21). on the contrary, The study that reported that $71.0 \%$ of patients were stage I, $17.2 \%$ were stage II, $9.4 \%$ were stage III, and $2.4 \%$ were stage IV, which indicates that patients presented in the early stage of disease(24).

\section{Conclusions}

Cancer larynx is generally a disease of elderly males, with a peak incidence in the fifth and sixth decade of life with 
low socioeconomic level and rural residence.

Tobacco is evidently the most important risk factor, It is commonly accepted that the risk is associated with the duration of exposure to this habit, but also the total intake dose of tobacco. Alcohol is the second predominant risk factor for development of laryngeal carcinoma. The results showed that the most affected laryngeal region was the glottic region and the most common histopathological type was SCC (97.10\%).

\section{References}

1-Jemal A, Siegel R, Ward E, Hao Y, Xu J, et al. Cancer statistics. CA Cancer J Clin.2008; 58:71-96.

2-Jemal A, Tiwari RC, Murray T, Ghafoor A, Samuels A, et al.Cancer statistics. CA Cancer J Clin. 2004; 54:8-29.

3-Parkin DM, Pisani P, Ferlay J.Estimates of the worldwide incidence of 25 major cancers in 1990. Int J Cancer. 2005; 80:827841.

4. Ballenger

JJ, Snow

JB.Otorhinolaryngology: head and neck surgery. 15th ed. Media, Williams \& Wilkins, PA, USA. 1996

5-Koufman J, Robert TS, Robert

T.Laryngopharyngeal reflux: consensus conference report. Journal of Voice. 1996; 10(3): 215-216.

6-Wünsch Filho V.The epidemiology of laryngeal cancer in Brazil. Sao Paulo Med J. 2004;122:188-194

7-Bray F, Ferlay J, Parkin DM, Pisani P.GLOBOCAN 2000 : Cancer incidence, mortality and prevalence worldwide. International Agency for Research on Cancer, Lyon. 2001;1:123-130.

8-Cattaruzza MS, Maisonneuve $\mathbf{P}$, Boyle P. Epidemiology of laryngeal cancer. Eur J Cancer B Oral Oncol. 1996; 32B:293-305.

9-Markou K, Christoforidou A, Karasmanis I, Tsiropoulos G, Triaridis S, Constantinidis I, et al. Laryngeal cancer: epidemiological data from Northern Greece and review of the literature. Hippokratia medical journal. 2013; 17(4): 313-318.

10-Rothman KJ.The effect of alcohol consumption on risk of cancer of the head and neck. Laryngoscope. 1978; 88:5155.

11-Rothman KJ.The effect of alcohol consumption on risk of cancer of the head and neck. Laryngoscope. 1978; 88:5155.

12- Maier H, Tisch M. Epidemiology of laryngeal cancer: results of the Heidelberg case-control study. ActaOtolaryngol. 1997; 527:160 164.

13-Menvielle G, Luce D, Goldberg P, Leclerc A.Smoking, alcohol drinking, occupational exposures and social inequalities in hypopharyngeal and laryngeal cancer. Int J Epidemiol. 2004; 33:799-806.

14-Lefebvre JL, Lartigau E, Kara A, Sarini J, Sobin LH.Oral Cavity, Pharynx, and Larynx Cancer. TNM Online. 2003

15-Boffetta P, Richiardi L, Berrino $F$, Estève $J$, Pisani $P$, Crosignani P.Occupation and larynx and hypopharynx cancer: an international case-control study in France, Italy, Spain, and Switzerland. Cancer Causes and Control. 2003; 14:203-212.

16-Muscat JE, Wynder EL.Tobacco, alcohol, asbestos, and occupational risk factors for laryngeal cancer. Cancer. 1992; 69:22442251. 
17-Zhang D, Jian Z, Bin C, Liang Z, Lei T.Gastroesophageal reflux and carcinoma of larynx or pharynx: a meta-analysis. Actaoto-

laryngologica . 2014; 134(10): 982989.

18-De Alba, Luz MD, Francisco MC. Risk factors for developing laryngeal cancer in adult population at the Hospital Espanol in Mexico City.ActaOncologica (English Edition). 2008; 59 (8): 367-370.

\section{9-Martínez O, Álvarez}

F.Epidemiología del cáncer de laringe en la provincia de Guadalajara Otorhinolaryngol DISP. 2002; 29:172-179.

20-Luna-Ortiz K, VillavicencioValencia V, Pasche $P$, LavinLozano A, Herrera-Gómeza Á.Laryngeal cancer in patients younger vs older than 40 years old: a matched-paired analysis. ActaOtorrinolaringolEsp 2010; 62:113-118.

21-AbdelEItawab HM, EIMessih MWA, AlNaggar NA, El
Sharkawy LS.Study of the epidemiology and management of laryngeal cancer in Kasr Al-Aini Hospital, Egypt J Otolaryngol.2015; 30:208-214.

22-De Stefani E, Boffetta P, DeneoPellegrini H, Brennan P, Oreggia F, Correa P, et al. Supraglottic and glottic carcinomas: epidemiologically distinct entities? Int $\mathrm{J}$

Cancer. 2004;112:1065-1071.

23-Myers EN, Suen JY, Myers JN, Hanna EY.Cancer of the head and neck.4th ed. Saunders, Philadelphia. 2003; 34: 340:370.16Muscat JE, Wynder EL.Tobacco, alcohol, asbestos, and occupational risk factors for laryngeal cancer. Cancer. 1992; 69:22442251.

24-Scola B, Fernandez-Vega M, Martinez T, Scola E, Ramirez C.The Gregorio Maranon Hospital experience with vertical partial laryngectomies. Arch Euroto-rhinolaryngol .1999; 256 (6): 296-298. 